\title{
What does the structure of liquid mean?
}

\author{
Takeshi Egami ${ }^{1,2}$ \\ ${ }^{1}$ University of Tennessee, Knoxville, United States of America; \\ ${ }^{2}$ Oak Ridge National Laboratory, Oak Ridge, United States of America; \\ egami@utk.edu
}

The structure of liquid and glass is usually described by the atomic pair-distribution function (PDF), $g(r)$, which expresses the statistical distribution of distances between atoms. The PDF can be determined by diffraction experiments using x-rays or neutrons. However, liquid is dynamic in nature, and we have to be sensible about what the PDF means for liquid. For crystalline solids the atomic structure is determined by the elastic scattering of $\mathrm{x}$-rays, neutrons or electrons, because the momentum is transferred to the whole rigid body of the sample in scattering. But the elastic scattering intensity from liquid is zero because of the lack of rigidity. The scattering from liquid is purely inelastic, described by the dynamic structure factor, $S(Q, \omega)$, where $Q$ is the momentum transfer and $E=h \omega / 2 \pi$ is the energy transfer in scattering. To measure $S(Q, \omega)$ we need an elaborate inelastic scattering instruments. In particular for inelastic $\mathrm{x}$-ray scattering (IXS) we need a very high energy resolution with $\sim \mathrm{meV}$ and $\Delta E / E<10^{-7}$. This can be achieved only with a large backscattering crystal analyzer with a long flight path. However, in regular x-ray diffraction measurement the energy resolution is poor, $\sim 1 \mathrm{eV}$, far exceeding the typical energies of vibrational excitations. As a result, the measured structure function, $S(Q)$, is the $S(Q, \omega)$ integrated over energy, thus representing the same-time correlation among atoms. Thus, the PDF, obtained by the Fourier-transformation of $S(Q)$, is the same-time density correlation function which shows the time averaged snapshot of correlations. Therefore, the PDF does not describe the structure in a regular sense. For a long time, the PDF has been used in representing the structure, because it was the only readily available structural descriptor, and various theories have been proposed to predict dynamic properties from the PDF. On the other hand, the dynamic two-body correlation can be directly expressed by the Van Hove function, $G(r, t)$, obtained by the double-Fourier-transformation of $S(Q, \omega)$. But, to carry out the double-Fourier-transformation accurately $S(Q, \omega)$ has to be measured over a wide $Q-E$ space. Until recently this was unpractical, because the inelastic scattering measurement, typically done with a triple-axis-spectrometer, was extremely time-consuming. But the advent of pulsed neutron sources with large two-dimensional detector arrays and advances in the IXS instrumentation made it possible to determine the Van Hove function in a reasonable time, $4-12 \mathrm{hrs}$. We applied this technique to various liquids, including water, aqueous solutions of salt, metallic alloy liquids, liquid Ga, and organic electrolytes. New physical insights obtained by these measurements will be discussed. Now that this technique is available we should expand the definition of the "structure" of liquid to include the dynamic structure represented by the Van Hove function.

Keywords: liquid, dynamics, inelastic scattering, structure 